\title{
AN INTELLIGENT MOBILE APPLICATION TO AUTOMATE FOOD HEALTH RECOMMENDATION USING DEEP LEARNING
}

\author{
Peiqi Gu${ }^{1}$, Yu Sun ${ }^{1}$ and Fangyan Zhang $^{2}$ \\ ${ }^{1}$ Department of Computer Science, California State Polytechnic University, \\ Pomona, CA, 91768 \\ ${ }^{2}$ ASML, San Jose, CA, 95131
}

\begin{abstract}
As the global health condition declines, people have started to be more conscious about their health. In addition, the development of deep learning, especially in the sector of image recognition, proliferates, provides more convenience for people to monitor their health. Even though some food recognition applications appear on the internet, most of them are inaccurate, and there aren't any researches that focus on the correlation between the accuracy of the model and attribute of the model. In addition, it is still inconvenient for people to gather information about how the food they eat everyday affects their health. Hence, in this project, the advanced development of deep learning was utilized for making an app which could be used to recognize a picture of the food taken by a phone and to display the food's effect on a person's certain health conditions. This project, or the application, has two main components: a model that can recognize the actual food through the camera of the phone and a database that stores the effects of the foods toward different kinds of health problems. After taking the photo, the application will display the effect of the foods to certain health problems that the user wants to see.
\end{abstract}

The experiment part of this project was inclined more on the optimization of the image recognition model. The result of this experiment indicated that more pictures in one category, less categories in total, and higher image resolution can improve the accuracy of the recognition model. This finding will be used on optimizing both the model and the application.

\section{KEYWORDS}

Deep Learning, Food Health Recommendation

\section{INTRODUCTION}

In the recent years, the global health condition declines as a side effect of the development of human technologies outruns the advancement of human body. However, one positive effect it leads to is that people start to care more about their health, and this results to a trend that there are more internet feeds about what kind of food leads to what kinds of outcome to a person's certain health conditions. Even though this type of information grows fast in quantity, many of them are low in quality.

At the same time, the development of artificial intelligence - especially the branch of deep learning (a sector of machine learning) ${ }^{[2]}$ - flourishes in this decade. Artificial Intelligence is the general category for all three of the terms, representing any intelligence displayed by a machine that is able to lead to an optimal or suboptimal solution given a problem. Machine learning, which

Natarajan Meghanathan et al. (Eds) : ACITY, AIAA, DPPR, CNDC, WIMNET, WEST, ICSS - 2019

pp. 31-36, 2019. (C) CS \& IT-CSCP 2019

DOI: $10.5121 /$ csit.2019.91703 
is a term created by Arthur Samuel in 1959, is defined as "the practice of using algorithms to parse data, learn from it, and then make a determination or prediction about something in the world." ${ }^{[3]}$ Deep learning ${ }^{[4]}$, a subcategory for machine learning, is inspired and imitates the

structure of a human brain, and like a brain's complex neutral structure, a deep learning model also contains layered structure, with each layer analyzing data, extracting useful information, and passing down the conclusion of the analysis to the next layer. Image recognition is the section of deep learning that making the greatest advancement in the recent years.

As a result, several health-related applications, such as Foodvisor ${ }^{[5]}$, emerge on the Internet that utilize AI to scan tangible foods. Though these apps encompass a wide range of food variety, the accuracy of such apps' scanning models is below expectation. Hence, there are still no efficient tools to gather health-related information, making it inconvenient for people who are interested in foods' effects toward human bodies to search up for information.

To solve this problem, Healthy Foodies, whose chief usage is to recognize the picture of the food taken by the phone and to display the food's effect on a person's certain health conditions, was done in a form of application, This application utilized the advanced development of deep learning, and it has two main components: a model that can recognize the actual food through the camera of the phone, and a database that stores the effects of the food toward different kinds of health problems. After taking the picture, the app will display the effect of the foods to certain health problems that the user wants to see. If this application can be carefully developed and widely spread, it would for sure have notable influence by making people's lives healthier and more convenient.

The central question of the research was how to improve the model's accuracy by manipulating the attributes of the algorithmic model. The hypothesis of this research was that the accuracy of the model is proportional to the quantity of the photo it used during the learning process, inversely proportional to the number of categories of food included in each model, and proportional to the quality of the photos being used in the learning process.

\section{Challenges}

To resolve the issues mentioned above, there are several impediments encountered throughout the development of the application and the implementation of the research. The first challenge is to build the deep learning model for the app. Before the development of the application, a model utilizing machine learning algorithms was planned to be devised. However, when considering that the algorithm of deep learning is more automatic in which it can "determine on [its] own if a prediction is accurate or not" ${ }^{[6]}$, deep learning is applied to this project. Yet, it would be too difficult to code an advanced deep learning model. Luckily, instead of developing one, a preexisting, free deep learning model from an online tutorial "TensorFlow For Poets" (which will be further explained in the following sections of this paper) is modified and implemented on the app. The second challenge is how to effectively test the accuracy of the model, and the solution is to use the Terminal application, which will be elaborated subsequently.

\section{METHODS AND MATERIALS}

A pre-existent model was utilized when constructing the recognition models that were used in this experiment. In the tutorial "TensorFlow For Poets" from Codelabs ${ }^{[7]}$ (a learning website run by Google), detailed steps of how to import, set up, and use the recognition model were presented. The model could be downloaded and imported by using the Terminal commands the tutorial provided. 
Terminal is a terminal emulator included in the macOS operating system. By typing commands in Terminal, the computer would execute certain actions. The following two lines were the commands for setting up the resolution limit of the input images and size of the model's structure.

\section{IMAGE_SIZE $=224$}

ARCHITECTURE="mobilenet_o.50_\$ $\{$ IMAGE_SIZE $\}$ "

After that, store the pictures of different types of food into different folders, with all folders were kept in a larger folder. This larger folder was then placed in the appointed folder, tf_files.

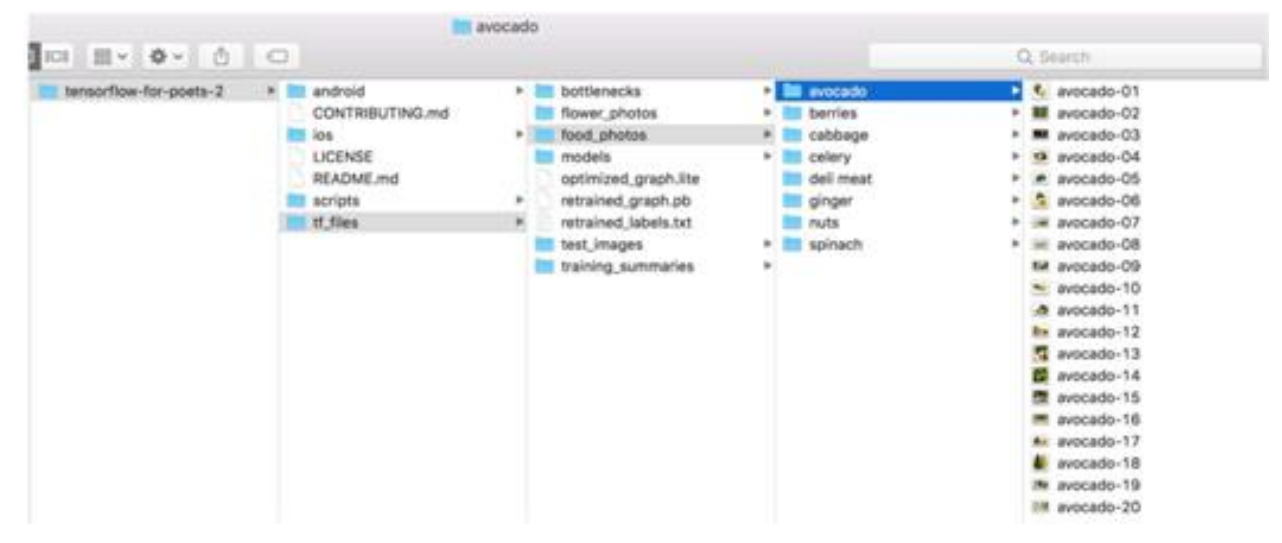

Figure 1: The structure of the tf_files

Once this process was done, use the set of commands below to start the training process for the model.

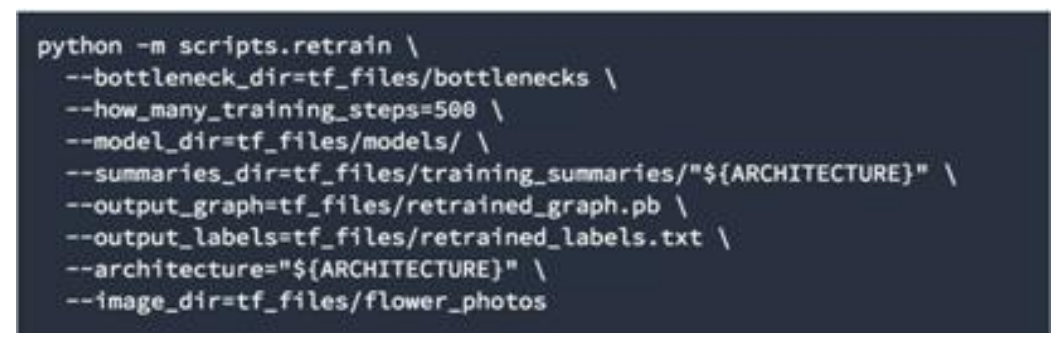

In this project, three independent variables were considered when constructing the model:

1. The quantity of picture samples for each food; $(25,50,75)$

2. The number of food categories stored in the model; $(2,5,8)$

3. The image resolution. $(160,192,224 \mathrm{px})$

Each independent variable would be tested in three scales (which are shown above after each variable) while the other two were held constant (the medium scale for each variable). One model would be created for each trial; therefore 7 testing models would be created eventually. Upon the construction of each model, each of them would scan 5 distinct photos that depict the same food. The average accuracy for each model in percentage would be recorded as the dependent variable of the research.

Since this experiment inclined more on the accuracy of the model but not the application, the accuracy of each model would be tested in Terminal. The first two independent variables could be varied by adjusting the files and folders in the tf_files folder. The last independent variable could 
be altered by changing the parameter of the IMAGE_SIZE commands when constructing the model.

The recognition models were tested in Terminal by using these following commands:

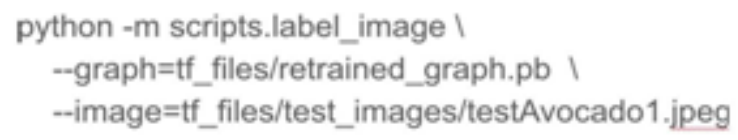

\section{RESUltS}

\section{Experiment 1: The Accuracy of the Recognition Model with Varied Quantity of Picture Samples for Each Food}

In this experiment, 3 trials were conducted. The quantity of picture samples for each food category were different in each trial $(25,50$, and 75 pictures in trial 1,2 , and 3 , respectively), while the number of food categories and image resolution were held constant at 5 categories and 192px, respectively.

Table 1. Data of experiment 1

\begin{tabular}{|l|l|l|l|l|l|l|}
\hline & $\begin{array}{l}\text { Trial 1 } \\
\text { Accuracy (\%) }\end{array}$ & Deviation & $\begin{array}{l}\text { Trial 2 } \\
\text { Accuracy (\%) }\end{array}$ & Deviation & $\begin{array}{l}\text { Trial 3 } \\
\text { Accuracy (\%) }\end{array}$ & Deviation \\
\hline Picture 1 & 82.87 & -3.96 & 89.11 & -4.46 & 92.82 & -3.68 \\
\hline Picture 2 & 87.43 & +0.60 & 94.20 & +0.63 & 96.55 & +0.05 \\
\hline Picture 3 & 84.05 & -2.78 & 93.29 & -0.28 & 96.17 & -0.33 \\
\hline Picture 4 & 91.53 & +4.70 & 96.24 & +2.67 & 98.64 & +2.14 \\
\hline Picture 5 & 88.26 & +1.44 & 95.03 & +1.46 & 98.30 & +1.80 \\
\hline Avg. & 86.83 & 2.70 & 93.57 & 1.90 & 96.50 & 1.60 \\
\hline $\begin{array}{l}\text { Percent } \\
\text { Deviation } \\
(\%)\end{array}$ & & 3.11 & & 2.03 & & 1.66 \\
\hline
\end{tabular}

\section{Experiment 2: The Accuracy of the Recognition Model with Varied Amount of food Categories}

In this experiment, 3 trials were conducted. The quantity of food categories was different in each trial $(2,5$, and 8 categories in trial 1,2 , and 3 , respectively), while the number of picture samples for each type of food and image resolution were held constant at 50 images and 192px, respectively.

Table 2. Data of experiment 2

\begin{tabular}{|l|l|l|l|l|l|l|}
\hline & $\begin{array}{l}\text { Trial 1 } \\
\text { Accuracy (\%) }\end{array}$ & Deviation & $\begin{array}{l}\text { Trial 2 } \\
\text { Accuracy (\%) }\end{array}$ & Deviation & $\begin{array}{l}\text { Trial 3 } \\
\text { Accuracy (\%) }\end{array}$ & Deviation \\
\hline Picture 1 & 98.97 & -0.34 & 89.11 & -4.46 & 85.74 & -4.17 \\
\hline Picture 2 & 99.41 & +0.10 & 94.20 & +0.63 & 89.55 & -0.36 \\
\hline Picture 3 & 99.23 & -0.08 & 93.29 & -0.28 & 88.14 & -1.77 \\
\hline Picture 4 & 99.52 & +0.21 & 96.24 & +2.67 & 94.21 & +4.30 \\
\hline Picture 5 & 99.44 & +0.13 & 95.03 & +1.46 & 91.89 & +1.98 \\
\hline Avg. & 99.31 & 0.17 & 93.57 & 1.90 & 89.91 & 2.52 \\
\hline $\begin{array}{l}\text { Percent } \\
\text { Deviation } \\
\%)\end{array}$ & 0.17 & & 2.03 & & 2.80 \\
\hline
\end{tabular}




\section{Experiment 3: The Accuracy of the Recognition Model with Varied Image Resolutions}

In this experiment, 3 trials were conducted. The image resolution was different in each trial (160px, 192px, and 224px in trial 1,2, and 3, respectively), while the quantity of picture samples for each type of food and the number of food categories were held constant at 50 images and 8 categories, respectively.

Table 3. Data of experiment 3

\begin{tabular}{|l|l|l|l|l|l|l|}
\hline & $\begin{array}{l}\text { Trial 1 } \\
\text { Accuracy (\%) }\end{array}$ & Deviation & $\begin{array}{l}\text { Trial 2 } \\
\text { Accuracy (\%) }\end{array}$ & Deviation & $\begin{array}{l}\text { Trial 3 } \\
\text { Accuracy (\%) }\end{array}$ & Deviation \\
\hline Picture 1 & 86.96 & -3.82 & 89.11 & -4.46 & 91.54 & -3.41 \\
\hline Picture 2 & 91.92 & +1.14 & 94.20 & +0.63 & 95.31 & +0.36 \\
\hline Picture 3 & 90.08 & -0.70 & 93.29 & -0.28 & 94.13 & -0.82 \\
\hline Picture 4 & 92.98 & +2.20 & 96.24 & +2.67 & 97.35 & +2.40 \\
\hline Picture 5 & 91.97 & +1.19 & 95.03 & +1.46 & 96.41 & +1.46 \\
\hline Avg. & 90.78 & 1.81 & 93.57 & 1.90 & 94.95 & 1.69 \\
\hline $\begin{array}{l}\text { Percent } \\
\text { Deviation } \\
(\%)\end{array}$ & & 1.99 & & 2.03 & & 1.78 \\
\hline
\end{tabular}

\section{RELATED WORK}

There are several researches and articles posted online that present information and procedures about recognition AI model training. One of such articles is "Train Image Recognition AI with 5 lines of code" [8] by Moses Olafenwa published on towardsdatascience.com. In this article, the author offers a series of steps of training one's own image recognition AI. According to the article, the given example model trained eleven categories with 900 images in each category. Then, 200 images from each category were tested. Although the AI model tend to be more accurate compared to my tested model, the article doesn't specifically address about the trend of accuracy of the recognition model and test multiple models that vary in their parameters.

\section{ANALYSIS AND DiSCUSSION}

All three points in the hypothesis were indicated in the data from the three experiments. The results were also logical because of the following reasons: with more images available, the model can be more "experienced", thus making its predictions more accurate; with more categories being stored, it will be harder for the model to distinguish between foods that have similar colors or textures; with photographs that have higher resolution, the model can analyze the structure and texture of the food more effectively, increasing its accuracy. However, there was a main drawback of this experiment that could cause a mislead in the data trend: inadequacy of trials (data points) for each experiment. For instance, in the first experiment, it should be true that the accuracy increases at a higher rate at the beginning and gradually slows down and eventually reaches a certain maximum, even though there was an indication in the decrement of the accuracy change,

since it only had three data points, the graph could also be interpreted as a linear trend, which should be false.

Hence, the experimental design could be improved by adding more trials for each experiment. In this way, the trend for each graph could be displayed. 


\section{CONClusion}

The original purpose of this project was to optimize the image recognition model so that the accuracy of predictions and the application could be optimized. Through a series of experiments by adjusting the model, it became apparent that with more picture samples, less categories, and higher image resolution, the recognition model would have the highest accuracy. As for the future work, I will explore more about deep learning and try to further improve the accuracy of the recognition model.

\section{REFERENCES}

[1] Arel, Itamar, Derek C. Rose, and Thomas P. Karnowski. "Deep machine learning-a new frontier in artificial intelligence research." IEEE computational intelligence magazine 5.4 (2010): 13-18.

[2] https://www.sas.com/en_us/insights/analytics/machine-learning.html

[3] https://becominghuman.ai/ai-machine-learning-deep-learning-explained-in-5-minutes- b88b6ee65846

[4] https://en.wikipedia.org/wiki/Deep_learning

[5] https://www.foodvisor.io/

[6] https://www.zendesk.com/blog/machine-learning-and-deep-learning/

[7] https://codelabs.developers.google.com/codelabs/tensorflow-for-poets/\#0

[8] https://towardsdatascience.com/train-image-recognition-ai-with-5-lines-of-code-8ed0bdd8d9ba 